Artículo de Investigación

Apuntes del CENES

ISSN 0120-3053

Volumen $34-\mathrm{N}^{\circ} .60$

julio - diciembre 2015

Págs. 95-130

\title{
Salarios, incentivos y producción intelectual docente en la universidad pública en Colombia*
}

\author{
Salaries, incentives and teaching intellectual \\ production in the public university in \\ Colombia
}

\author{
Salários, incentivos e produção intelectual \\ docente na universidade pública \\ em Colômbia
}

Jhon Alexánder Méndez Sayago** Leonardo Vera Azaf***

Fecha de recepción: 3 de enero de 2015

Concepto de evaluación: 8 de abril de 2015

Fecha de aprobación: 25 de junio de 2015

\footnotetext{
Este artículo forma parte de la tesis de grado "Salarios y productividad laboral en Colombia" para optar por el título de Doctorado en Economía del Desarrollo de la Facultad Latinoamericana de Ciencias Sociales sede Ecuador.

** Profesor asistente, Departamento de Economía, Universidad del Valle, Cali, Colombia. Ingeniero civil de la Universidad Francisco de Paula Santander. Máster en Economía de la Universidad Javeriana. Máster en Economía del Medio Ambiente y de los Recursos Naturales de la Universidad de los Andes. Correo electrónico: Jhon.mendez@correounivalle.edu.co

*** Profesor asociado, Escuela de Economía, Universidad Central de Venezuela, Caracas, Venezuela. Ph.D. Universidad de Londres, Inglaterra. Correo electrónico: leoverave@gmail.com
} 
Salarios, incentivos y producción intelectual docente en la universidad pública ...

Jhon Alexánder Méndez Sayago - Leonardo Vera Azaf

\title{
Resumen
}

En este artículo se estudian los determinantes de la productividad intelectual de los profesores de la universidad pública en Colombia, enfocándose en el efecto del incentivo salarial por productividad intelectual y el efecto salario. A partir de información del cuerpo de profesores de la Universidad del Valle, comprendida entre los años 2004-2012, se estimaron modelos econométricos de ciclo de vida del profesor universitario para cuantificar dichos efectos. Se encontró un efecto importante y significativo del valor presente del punto salarial, pero no se halló efecto positivo del salario sobre la producción intelectual. También se calculó el efecto del incentivo en función de la edad del investigador y el impacto de incrementos porcentuales en el valor del punto salarial.

Palabras clave: producción intelectual, modelo de ciclo de vida, profesor universitario, modelo Tobit, incentivo.

JEL: C24, J24

\begin{abstract}
In this article the determinants of intellectual productivity of teachers of public universities in Colombia are studied, focusing on the effect of wage incentive on intellectual productivity and wage effects. Based on information from the teaching staff of the Universidad del Valle between the years 20042012, an econometric model lifecycle university professor were estimated to quantify these effects. An important and significant effect of the present value of the salary point was found, but no was found positive effect of wages on intellectual production. The effects of the incentive depending on the age of the researcher and the impact of percentage increases in the value of the salary point were also calculated.
\end{abstract}

Keywords: intellectual production, life cycle model, professor, Tobit model, incentive. 


\section{Resumo}

Este artigo discute os determinantes da produtividade intelectual de professores de universidades públicas na Colômbia, concentrando-se sobre o efeito da produtividade intelectual salário de incentivo e efeito salário são estudados. A partir de informações do corpo docente da Universidad del Valle, entre os anos de 2004-2012, professor econométrico modelos de ciclo de vida universitária para quantificar esses efeitos foram estimados. Um efeito importante e significativo do valor presente do ponto de salário foi encontrado, mas nenhum efeito positivo dos salários foi encontrado na produção intelectual. O efeito do incentivo, dependendo da idade do investigador e do impacto de percentagens de aumento no valor do ponto de salário também foi calculada.

Palavras-chave: produção intelectual, modelo de ciclo de vida, professor universidade, modelo Tobit, incentivo. 
Salarios, incentivos y producción intelectual docente en la universidad pública ... Jhon Alexánder Méndez Sayago - Leonardo Vera Azaf

\section{INTRODUCCIÓN}

Tal como lo señalan Baccini, Barabesi, Cioni y Pisani (2014) y Obembe (2012), el estudio de la productividad científica y de los factores asociados a ella ha sido un tema que ha atraído la atención de los investigadores especialmente en los últimos treinta años.

Stephan (2010) expone tres razones por las cuales la economía de las ciencias ha venido ganando terreno en los últimos años. En primer lugar, la ciencia se ha identificado como una fuente de crecimiento, lo que se ha corroborado por los avances recientes en las tecnologías de la información, situación que contribuyó de manera significativa al crecimiento en los sectores de servicios en los últimos años. Los avances en la investigación médica también han llevado a una gran expansión del trabajo y la esperanza de vida. La segunda razón tiene que ver con el tema de la apropiabilidad del conocimiento. Es decir, una vez el conocimiento se produce y se hace público, no se puede excluir a los otros de su consumo. Por lo tanto, el fracaso de las economías al tratar de producir la cantidad óptima de conocimiento considerándolo como un bien público, es un tema importante para los economistas. Por último, el carácter público de la investigación y el spillover inherente en un sistema de estas características, resulta fundamental para el concepto de la teoría del crecimiento endógeno (Archibugi \& Coco, 2004).

Perozo, Arteaga y Fuenmayor (2008) argumentan que la investigación se ha constituido en el proceso que más contribuye al desarrollo de los países del mundo, lo que explica que las grandes potencias inviertan altos porcentajes de 
su producto interno bruto (PIB) en investigación y desarrollo, logrando con esto posicionarse en los grandes adelantos científicos y tecnológicos. Esta situación lleva a afirmar que la investigación es reconocida a nivel mundial como un pilar de la consecución de nuevos conocimientos y tecnologías. Esta compleja actividad involucra empresas, instituciones, universidades nacionales y redes internacionales de colaboración para alcanzar mejores resultados.

Las exigencias actuales de la sociedad también obligan a que los profesores, desde diversas posiciones científicas, busquen soluciones concretas a los problemas que demanda el contexto educativo en el cual se desempeñan. Surge de allí la idea de que las universidades deben concebirse como centros de producción intelectual, tomando en cuenta que en el ámbito académico, la investigación es la que genera conocimiento. En la sociedad del conocimiento se hace indispensable la generación de nuevos saberes, y es el profesor universitario quien está llamado a producir y a revisar los mismos. En efecto, el resultado de las investigaciones ayuda a mejorar la praxis docente de forma permanente y por ende, la investigación representa, una actividad de primer orden en las universidades.

Ante estos cambios, las universidades colombianas tradicionalmente enfocadas en la docencia, cambiaron su visión y desarrollaron políticas pensando en la investigación como una de sus prioridades, especialmente las universidades públicas y las universidades privadas más reconocidas, ofreciendo incentivos a las publicaciones y buscando que a través de estas, los profesores generen conocimiento $\mathrm{y}$ lo divulguen en revistas indexadas nacionales e internacionales, libros de investigación, o participen en congresos y eventos académicos (Guzmán \& Trujillo, 2011).

En dicha política de incentivos se pueden identificar básicamente dos estrategias. La primera son salarios de partida relativamente bajos que se incrementan en función de la producción intelectual. En la segunda se ofrecen salarios iniciales relativamente más altos y primas por producción intelectual durante un periodo limitado, generalmente un año.

Uno de los principales problemas a los que se enfrenta la universidad pública en Colombia en la contratación de nuevos profesores, son las restricciones legales que afronta para fijar un salario de enganche que pueda atraer a los candidatos más talentosos del mercadoı. No obstante, también la universidad pública ofrece ventajas para que profesores con habilidad investigativa deseen incorporarse a la universidad pública: 1) no exige dedicación exclusiva, 2) dependiendo de su antigüedad, experiencia y sobre todo

En el año 2014 el salario promedio de enganche de un profesor con doctorado fue de apenas 3,8 millones de pesos en promedio. 
publicaciones, un profesor de universidad pública puede llegar a ganar tanto o más que un profesor de universidad privada.

El problema es que una vez contratado el nuevo profesor, dado su bajo salario, podría optar por las otras actividades generadoras de ingreso, en perjuicio del tiempo dedicado a la investigación, lo que afectaría la producción intelectual que es uno de los objetivos de la dirección universitaria porque esto le da prestigio a la institución.

Este artículo trata de establecer cuál de las dos alternativas es más efectiva como instrumento para el estímulo de la producción intelectual docente. En búsqueda de este objetivo, este artículo investiga los determinantes de la productividad intelectual de los profesores de la universidad pública en Colombia, enfocándose en el efecto del incentivo salarial por productividad intelectual $\mathrm{y}$ el efecto salario.

Para comprender la relación entre salario, incentivo salarial y producción intelectual, en el artículo se hace una adaptación en tiempo discreto del modelo de ciclo de vida de Levin y Stephan (1991). La innovación consiste en que el salario futuro del profesor investigador depende de las publicaciones durante todo su ciclo de vida, y no de su prestigio.

Posteriormente, a partir de información del cuerpo de profesores de la Universidad del Valle comprendida entre los años 2004-2012, se estimó econométricamente el modelo de ciclo de vida del profesor universitario para cuantificar los efectos del salario y el incentivo salarial sobre la producción intelectual.

El artículo está organizado de la siguiente forma: en la primera sección se presenta una revisión el estado de la cuestión, que da cuenta de los principales determinantes de la producción científica y se reseñan modelos teóricos de ciclo de vida que tratan de explicar la dinámica de la productividad del investigador a lo largo del tiempo. En la segunda sección se analiza desde la teoría del agente principal la relación contractual entre la universidad pública y el profesor universitario, con énfasis en los incentivos para el estímulo de la producción intelectual. En la sección tres se desarrolla un modelo de ciclo de vida para los profesores de la universidad pública en Colombia, que desvela el impacto del salario y el incentivo salarial sobre su producción intelectual. En la sección cuatro se presentan los datos y sus estadísticas descriptivas, en la sección cinco se realiza la estimación econométrica del modelo de ciclo de vida, en la sección seis se analizan los resultados, para finalmente presentar las conclusiones.

\section{REVISIÓN LITERARIA}

Print y Hattie (1997) definen la producción científica como el conjunto de investigaciones desarrolladas por académicos en las universidades y contextos relacionados en un determinado periodo de tiempo. Para 
Piedra y Martínez (2007), la producción científica es considerada como la parte materializada del conocimiento generado, por eso, muchos se refieren a ella como el resultado en forma de publicaciones de los trabajos de investigación, como por ejemplo artículos publicados en revistas internacionales, locales o nacionales, los libros y capítulos de libros, las ponencias en conferencias, los documentos de trabajo, etc.

Otros sitúan la producción científica en un ámbito más amplio que la simple publicación. Según dicho punto de vista, «las tesis defendidas y que aún no han sido publicadas, los trabajos presentados en congresos, coloquios y simposios, aulas, trabajos de laboratorios concluidos y no publicados, incluso trabajos de campo; todo eso es producción científica» (Piedra \& Martínez, 2007, p. 3).

Aun reconociendo que la productividad científica engloba la totalidad de la investigación producida por los científicos, su elemento medible es la cantidad de publicaciones que produce un autor, una institución o un país determinado. Manjarrés (2009) expone argumentos como la revisión por pares de Fielden y Gibbons (1991), las preferencia de los investigadores de Crane (1965) y Merton (1968), la homogenización de Paasi (2005), la percepción del prestigio de la revista de Miller y Serzan (1984), para concluir que el uso del número de artículos publicados por el profesor en revistas internacionales es la principal medida de la producción científica de la actualidad.
Al respecto, en Colombia profesores universitarios y editores de revistas se han manifestado en contra de esa forma de socialización del conocimiento impuesta desde estándares de la llamada ciencia de centro que conduce a una fuerte invisibilización de la producción académica colombiana y de sus publicaciones científicas.

Posterior a la discusión del concepto de productividad científica, se puede comenzar a explorar el estado del arte de las distintas formas de análisis la productividad científica. Baccini et al. (2014) distinguen dos aproximaciones alternativas: la primera se centra en las leyes fundamentales de la distribución de frecuencia del número de publicaciones (o citas), mientras que la segunda tiene como objetivo identificar los determinantes de la producción intelectual.

Baccini et al. (2014) también mencionan que la primera aproximación tiene origen en el estudio pionero de Lotka (1926), sobre la distribución de la frecuencia del rendimiento científico de químicos y físicos. Lotka concluye que el $60 \%$ de estos individuos realiza una sola contribución a lo largo de su vida, de forma tal que el número de autores con contribuciones es de aquellos que hacen solo una.

Baccini et al. (2013) además citan argumentos de otros investigadores que pretenden dar cuenta de las diferencias entre los investigadores: 
Salarios, incentivos y producción intelectual docente en la universidad pública ...

Jhon Alexánder Méndez Sayago - Leonardo Vera Azaf

El denominado sacred-spark hypothesis propuesto por Allison y Stewart (1974) y David (1994), según el cual las diferencias en la productividad reflejan capacidades desiguales y predeterminadas de los investigadores.

La llamada Matthew-effect hypothesis desarrollada por Merton (1968), la cual señala que los investigadores de renombre tienen mayor facilidad para publicar sus trabajos que otros investigadores menos conocidos, a pesar de que estos cuenten con contribuciones de calidad equivalente

Cole y Cole (1973) plantearon la cumulative advantage hypothesis. La idea es que el reconocimiento recibido en una etapa temprana de la carrera de los investigadores puede ser reforzado con el tiempo, ya que esto le permite más fácil acceso a los recursos para la investigación. Lo que significa que cualquier ventaja será acumulativa.

La segunda aproximación dirige su atención a la identificación de los determinantes individuales y colectivos de la productividad científica. Los primeros se refieren a los atributos individuales del investigador (género, edad, disciplina científica, etc.) y los segundos son factores relacionados con el entorno en el que se desenvuelve el docente y el sistema general de recompensa de la ciencia. Manjarrés (2009) distingue entre el entorno académico y el socioeconómico, en este último se considera el efecto de la financiación procedente de agentes industriales, y en general las relaciones universidad-empresa.

El objetivo de esta sección es revisar la literatura relacionada con los determinantes de la productividad intelectual, explorando los enfoques basados principalmente en el estudio de las características individuales y del entorno académico del docente, porque en Colombia las relaciones entre la universidad pública y las empresas es muy débil. Como manifiestan Gutiérrez y Berrío (2011), la realidad de la relación en Colombia es cada quien en lo suyo.

\section{Factores individuales}

La bibliografía sobre los determinantes de la productividad científica de los docentes universitarios incluye como variables explicativas características individuales de los académicos tales como la edad, el género, la posición dentro de la institución, y la disciplina científica. La relación entre la edad y la productividad científica es el aspecto que más ha llamado la atención de economistas y sociólogos, dando origen a los denominados modelos de ciclo de vida de los investigadores.

Aunque los resultados obtenidos han sido diversos, muchos estudios indican la existencia de una relación no lineal, en forma de $U$ invertida, entre la edad y la productividad de los investigadores (Bayer \& Dutton, 1977; Cole, 1979; Zuckerman \& Merton, 1972; Weiss \& Lillard, 1982; Levin \& Stephan, 1991; 
González \& Veloso, 2007). También se ha señalado que el pico de productividad es heterogéneo, varía en función del campo disciplinar del docente (Levin \& Stephan, 1989). Lehman (1958, 1960) encontró que los científicos de las ciencias duras alcanzan su pico de productividad más pronto que los que pertenecen a otras disciplinas. Otros han hallado que la curva de productividad tiene dos picos (Bayer \& Dutton, 1977). Por otra parte, Allison y Stewart (1974) notaron correlación positiva entre la desigualdad en la productividad y los grupos de edad, de manera que a mayor edad, mayores diferencias entre los investigadores.

Los primeros estudios sobre factores determinantes de la productividad científica se basaron en datos de corte transversal, y pretendían explicar la productividad acumulada durante un periodo determinado de tiempo. Sin embargo, los resultados son cuestionados por la imposibilidad de distinguir entre el supuesto efecto de la edad y el que correspondería a la cohorte generacional. Posteriormente, la disponibilidad de datos longitudinales permite analizar la productividad de los investigadores de una misma cohorte con edades distintas, mientras envejecen, pudiendo así separar los dos efectos (Manjarrés, 2009).

Rauber y Ursprung (2008), a partir información de economistas académicos alemanes, encuentran diferencias entre las diferentes cohortes, en la relación entre la edad y la productividad intelectual. En los investigadores de mayor edad, la productividad se mantiene a lo largo de su vida, mientras que en los jóvenes se observa una forma de $\mathrm{U}$ invertida bastante pronunciada.

Analizando los determinantes de la productividad observada durante el periodo 1995-2000 de una muestra con 1.134 investigadores de la Universidad Louis Pasteur de Francia, Carayol y Matt (2006) encontraron a partir de una regresión Tobit, que la edad afecta negativamente la productividad, pero rechazan la forma de U invertida.

Debido a la importancia que tiene el factor de la edad en la explicación de la productividad académica para esta investigación, se dedica esta sección para la revisión de los principales modelos económicos de ciclo de vida que ayudan a comprender dicha relación.

La experiencia profesional también se puede considerar como un factor que afecta la producción intelectual. Rebne (1990) y Goodwin y Sauer (1995) concluyen que el rendimiento máximo de un investigador ocurre dentro de los primeros diez años de trabajo y tiende a disminuir después de 25 o 30 años de actividad académica.

También se ha indicado que la posición que ocupa el docente dentro de la institución puede llegar a ser un factor determinante para explicar la productividad científica. Knorr, Mittermeier, Aichholzer y Waller (1979) hallaron que cuando se controla el efecto 
Salarios, incentivos y producción intelectual docente en la universidad pública ...

Jhon Alexánder Méndez Sayago - Leonardo Vera Azaf

que ejerce la realización de labores administrativas, la edad deja de ser un factor significativo.

Muchos estudios han demostrado que la productividad intelectual tiende a aumentar dependiendo de la jerarquía del individuo en los puestos académicos. Manjarrés (2009) cita que Cole y Cole (1973), Long (1978) y Carayol y Matt (2006) encontraron que los profesores con mayor categoría dentro de la institución, como por ejemplo docentes de tiempo completo, tienen una mayor productividad que los profesores de categorías inferiores (junior o asistente).

Un estudio de Aksnes, Rorstad, Piro y Sivertsen (2011) indicó que los profesores de tiempo completo son los más productivos. En promedio, los profesores varones publicaron 9,5 publicaciones durante un período de cuatro años, seguido por profesores asociados 4,8 publicaciones y los doctores 4,5 publicaciones, mientras que los estudiantes de doctorado tienen la productividad más baja (2,9 publicaciones). Respecto a esas diferencias, Kyvik (1991) halló que los profesores, por ser líderes de grupos de investigación, aparecen en todas las publicaciones, mientras que los estudiantes de doctorado solo aparecen en las de su autoría.

Se debe tener en cuenta que la incorporación de variables, como el rango académico o la experiencia profesional, puede generar problemas de multicolinealidad con la variable edad, minando su capacidad explicativa o alterando el sentido de su influencia (Carayol \& Matt, 2006).

Otro atributo personal asociado con la productividad científica es el género. Muchos estudios han encontrado grandes diferencias de género en la productividad intelectual a favor de los hombres (Aksnes et al., 2011b; Kyvik \& Teigen, 1996; Cole \& Zuckerman, 1984). Por ejemplo, Aksnes et al. (2011b) encontraron que para casi todos los grupos de edad, los hombres son más productivos que las mujeres. Los científicos de sexo femenino tienden a publicar generalmente entre $20-40$ por ciento menos que sus colegas masculinos. Para explicar dicha diferencia, se han realizado investigaciones que incluyen la variable estado civil para tratar de explicar la productividad intelectual. La hipótesis es que las investigadoras casadas podrían ser menos productivas dadas sus responsabilidades domésticas. Sin embargo, la evidencia con respecto a este punto es ambigua (Reskin, 1978; Astin \& Bayer, 1979).

El campo de estudio es otro de los factores clásicos que pueden determinar la productividad científica. Dundar y Lewis (1998) hallaron diferencias significativas en el número promedio de artículos publicados por los académicos de universidades de Estados Unidos en función de la disciplina académica a la que pertenecen. Ellos encontraron que mientras que un docente representativo en el campo de las ciencias sociales 
publicó aproximadamente 2,5 artículos entre 1988 y 1991, sus colegas de biología publicaron 9 artículos durante el mismo periodo. Sin embargo, los autores señalaron que estos resultados reflejan más las diferencias generales en las pautas de publicación existentes entre diversas áreas, que auténticas diferencias en cuanto a los niveles de actividad investigativa por disciplina.

\section{Factores institucionales}

Los factores institucionales también surgen como posibles determinantes de la producción científica. Estos factores están relacionados principalmente con la institución, la facultad o el departamento al que se encuentra vinculado el docente, o el grupo donde el científico desarrolla su trabajo investigativo. De acuerdo con Manjarrés, (2009, p. 62):

Las políticas y objetivos institucionales son el marco de referencia que subyace en los procesos de evaluación del rendimiento científico y el fundamento de los sistemas de recompensa de la ciencia. En este sentido, los rasgos distintivos entre las políticas, objetivos o el énfasis de las misiones universitarias, pueden generar culturas organizativas diferentes, las cuales pueden incidir sobre la productividad científica del docente.

Para medir la influencia de las características de la institución o el departamento sobre la productividad intelectual,
Manjarrés (2009) cita a Kyvik (1995), Bonaccorsi y Daraio (2003), Smeby y Try (2005), como autores que han incluido en sus modelos econométricos variables como el tamaño del departamento. Jordan, Meador y Walters (1989) han tomado en cuenta el carácter público o privado de la institución, Creswell (1986) y Long (1978), el prestigio del departamento o de la institución.

Se supone que las organizaciones más grandes pueden acumular más recursos para la investigación que aumentan la productividad. Estos efectos acumulativos pueden acompañarse además de economías de escala en la producción científica. Los resultados no son concluyentes, mientras algunos estudios encuentran una relación positiva (Dundar \& Lewis, 1998), otros encuentran escasa relación (Cohen, Florida \& Goe, 1994) o solo en algunas disciplinas como las ciencias naturales (Kyvik, 1995).

La relación entre la carga docente y la productividad no está claramente determinada, mientras que en algunos estudios aparece una relación negativa en términos del costo de oportunidad (Fox, 1992) en otros estudios esta resulta positiva (Dundar \& Lewis, 1998; Kyvik \& Smeby, 1994).

\section{Técnicas de estimación y unidad de análisis}

En la literatura se emplean diferentes métodos de estimación. Al tratarse la productividad de datos de recuento, 
Salarios, incentivos y producción intelectual docente en la universidad pública ...

Jhon Alexánder Méndez Sayago - Leonardo Vera Azaf

la mayoría de los estudios opta por métodos como el de Poisson, el binomial negativo $\mathrm{y}$, ante proporciones muy elevadas de ceros en la variable dependiente, el modelo Hurdle. También se utiliza el método MCO con la variable dependiente en logaritmos y el modelo Tobit.

La unidad de análisis más frecuente en el análisis de la productividad intelectual es la individual, pero también pueden identificarse estudios a nivel de grupo de investigación, departamento, facultades o incluso territorios (Smeby \& Try, 2005; Bonaccorsi \& Daraio, 2003; Dundar \& Lewis, 1998).

Porter y Umbach (2001) demuestran que el efecto de la edad en un modelo que emplea técnicas multinivel difiere significativamente de los ofrecidos por un modelo de análisis a nivel individual. Smeby y Try (2005) encuentran que la edad de los investigadores tiene un efecto negativo sobre la productividad a nivel individual, pero encuentran que la edad media de los departamentos tiene efectos positivos en la productividad a nivel de grupo. El argumento que utilizan para defender sus resultados es que los investigadores de mayor edad asumen tareas de apoyo a la investigación, como organización de seminarios o motivación y supervisión de jóvenes investigadores que generan importantes externalidades. Además como la reputación está basada en las publicaciones realizadas y en los contactos profesionales, los investigadores de mayor edad tienen mayor capacidad para atraer financiación y colaboraciones prestigiosas, y a los jóvenes investigadores con mejores perspectivas.

\section{SALARIOS E INCENTIVOS PARA LA PRODUCCIÓN INTELECTUAL EN LA UNIVERSIDAD PÚBLICA EN COLOMBIA}

El contrato es un conjunto de reglas que facilita la cooperación y el intercambio realizado por agentes económicos racionales, porque crea los incentivos necesarios para que los agentes realicen las transacciones. En cualquier interacción económica se pueden identificar dos partes: el principal y el agente. EI principal es quien contrata al agente para realizar un trabajo. Los empleados (agente) se comprometen a usar sus habilidades para realizar las tareas que el empleador (principal) les ordene hacer, y el empleador se compromete a pagar a los empleados y a mantener un ambiente laboral favorable (Gorbaneff, 2003).

El modelo del agente principal permite abordar el análisis de las políticas de incentivos relacionados con la investigación en la universidad pública en Colombia. En el contrato laboral que pactan la universidad (principal) y los docentes de carrera (agentes), estos se comprometen a desarrollar actividades de investigación, docencia y extensión. El contrato incluye una remuneración determinada, y especifica un nivel de esfuerzo que es explícito en la carga académica del docente, en términos 
de las horas que él se compromete a dedicar a la docencia, la investigación y la extensión. El principal no puede observar directamente las acciones del agente(tiempo efectivo dedicado a la investigación), así que el cumplimiento del docente se verifica mediante productos que el profesor entrega al final del periodo académico (Carvajalino \& Ariza, 2008).

La universidad se beneficia cuando el profesor publica su producción intelectual, porque esto le da visualización y status. No obstante, no hay mecanismos de control efectivos que le permitan a la universidad influir directamente sobre el nivel de esfuerzo del profesor, en cuanto a la calidad de su producción intelectual o su esfuerzo por lograr la visualización de su trabajo. Esto genera la necesidad de un esquema de incentivos que logren que el profesor desarrolle el máximo esfuerzo en investigación, de forma tal que los resultados favorezcan a la universidad y al docente.

El Decreto 1279 de junio de 2002 es la norma que determina los ingresos salariales y estímulos académicos de la carrera profesoral. Por tanto, establece los incentivos a la producción intelectual de los profesores de la universidad pública en Colombia. De acuerdo a esta normatividad vigente, el salario de los profesores es el resultado de multiplicar el valor del punto salarial () y la cantidad de puntos salariales acumulados que se le hayan reconocido al profesor.
El valor del punto es determinado cada año por el Gobierno nacional, y la cantidad de puntos depende de la valoración de los siguientes factores:
a. La categoría dentro del escalafón docente.
b. Títulos de estudios universitarios.
c. La experiencia calificada.
d. La productividad académica.
e. Las actividades de dirección académico-administrativas.
f. El desempeño destacado en labores de docencia y extensión.

Las categorías que define el Decreto 1279 para el escalafón docente son: profesor auxiliar, profesor asistente, profesor asociado y profesor titular. A medida que asciende en el escalafón docente, el profesor va ganando puntos salariales. Un profesor titular alcanza el máximo de 96 puntos. Los docentes ascienden en el escalafón teniendo en cuenta las capacidades intelectuales, se le reconocen los méritos alcanzados por escolaridad, experiencia docente calificada y la producción intelectual en el campo profesional o del saber en el que se desempeña.

La determinación de la remuneración de los docentes universitarios también se basa en la formación, con esto se persigue estimular al docente a no estacionarse en un nivel educativo y pretende recompensar las inversiones que los docentes hacen en el capital humano, en el desarrollo intelectual. Por ejemplo, un profesor con maestría 
Salarios, incentivos y producción intelectual docente en la universidad pública ...

Jhon Alexánder Méndez Sayago - Leonardo Vera Azaf

puede recibir 80 puntos salariales por obtener su título de doctorado y hasta 10 puntos adicionales por cada estudio de posdoctorado.

La valoración de la experiencia calificada del Decreto 1279 otorga más puntos cuando un candidato ha trabajado en instituciones de investigación ciencia, técnica, humanidades, pedagogía (37 puntos por año) que cuando ha sido profesor en una universidad (4 puntos por año).

La producción intelectual del profesor es valorada de dos formas diferentes, mediante puntos salariales y con el reconocimiento de bonificaciones.

La valoración de los puntos salariales es fijada por el artículo 10 del Decreto 1279. La publicación de artículos en revistas tipo $A 1=15$ puntos, en revistas tipo $\mathrm{A} 2=12$ puntos, en revistas tipo $\mathrm{B}=8$ puntos, en revistas tipo $\mathrm{C}=3$ puntos, según el sistema de indexación de Colciencias. La producción de videos, cinematográficos o fonográficos de impacto internacional, 12 puntos, de impacto nacional, 8 puntos. La publicación de libros que resulten de una labor de investigación, hasta 20 puntos. La publicación de libros de texto hasta 15 puntos. La publicación de libros de ensayo, hasta 15 puntos por cada libro. Traducción de libros, hasta 15 puntos. Premios nacionales e internacionales, hasta 15 puntos. Patentes, hasta 25 puntos. Producción técnica, hasta 15 puntos por innovaciones, 8 puntos por adaptaciones. Producción de software, hasta 15 puntos.
Los puntos salariales por docencia destacada se otorgan teniendo en cuenta la evaluación del docente realizada por los estudiantes. El desempeño destacado en labores de extensión se hace con base en un documento en el cual el docente sustente la presencia de la universidad ante la comunidad, la relevancia académica y social del servicio, su complejidad y singularidad y una evaluación de la actividad por parte de la comunidad o institución beneficiadas. Los puntajes salariales anuales que se asignan a los docentes destacados en docencia y extensión son: profesor titular (hasta 5 puntos), profesor asociado (hasta 4 puntos), profesor asistente (hasta 3 puntos), profesor auxiliar (hasta 2 puntos).

Queda claro que el Decreto 1279 contiene un sistema de incentivos para el fomento de la producción intelectual de los docentes de carrera de la universidad pública. Este sistema de incentivos premia con puntos salariales la producción intelectual que le da mayor visibilidad a la universidad. El monto del incentivo económico corresponde al valor presente neto del valor del punto salarial.

Guzmán y Trujillo (2011) concluyen que los incentivos para la investigación tienen efecto principalmente sobre los profesores jóvenes, recién vinculados con vocación media para la investigación, para los cuales el costo de su esfuerzo por investigar debe ser cubierto por el incentivo a la investigación, $y$ así evitar que se incline únicamente por las actividades de docencia y reparta su 
esfuerzo entre ambas actividades, a un costo asumible para la sociedad.

Carvajalino y Ariza (2008) desarrollaron una encuesta a los docentes de la Universidad Industrial de Santander, para captar la percepción de los profesores sobre la eficacia de los incentivos para la investigación y su motivación en general.

La encuesta mostró que la mayoría de los docentes consideran que la motivación para pertenecer a un grupo de investigación se da por la posibilidad de desarrollo y reconocimiento académico personal $(70.73 \%)$. Otra motivación es ascender en el escalafón docente para el cual más de la mitad de los docentes encuestados le asignaron una alta valoración. Un $65.85 \%$ de los docentes consideran que es de gran motivación resolver un puzzle. Por último, el $24.39 \%$ de los docentes consideran que la principal motivación es conseguir aumentos salariales o bonificaciones. La encuesta dejo ver que el incentivo económico no es la razón principal para que el docente investigue. La posibilidad de desarrollo y reconocimiento personal, así como mejorar su escalafón docente son argumentos que apoyan la hipótesis de que profesor investiga porque esto mejora su estatus dentro de la universidad.

En la misma encuesta un $14.63 \%$ de los docentes considera que la Universidad incentiva la investigación con aumentos salariales, un $19.51 \%$ de los docentes supone que incentiva por medio de bonificaciones, un $9.76 \%$ de los docentes cree que incentiva por medio de la distribución de la carga académica, un 26.83 $\%$ de los docentes piensa que incentiva por medio de distinciones académicas, un $34.15 \%$ de los docentes considera que incentiva por medio de comisiones de estudio.

La encuesta reveló que menos del $10 \%$ de los profesores encuentra que la asignación de las horas de investigación de la carga académica es un incentivo para la investigación, es decir, que ese mecanismo de regulación directa para la investigación no funciona, de acuerdo con el mismo agente (profesor).

\section{MODELO DE CICLO DE VIDA PARA LOS PROFESORES DE LA UNIVERSIDAD PÚBLICA EN CO- LOMBIA}

Los modelos de ciclo de vida del investigador científico, son modelos de optimización intertemporal de la utilidad o el ingreso del investigador, que relacionan el tiempo dedicado a la investigación, y por tanto, sus resultados de investigación, con la edad. Como el stock de conocimiento del investigador que le da prestigio, depende del tiempo dedicado a la investigación, en estos modelos también se obtiene de forma derivada la relación entre dicho stock de conocimiento y la edad. Se pueden destacar, entre otros, el artículo pionero de Diamond (1984), y los aportes de Levin y Stephan (1991) y Rauber y Ursprung (2005). En términos generales, los modelos de ciclo de vida citados revelan por 
qué variables como el retorno marginal de los productos de investigación, la utilidad marginal de resolver un puzzle científico, las tasas de aprendizaje y de depreciación del conocimiento, afectan la producción intelectual del investigador y por qué declina esta con la edad.

En esta sección se formula un modelo de ciclo de vida para los profesores de la universidad pública en Colombia, asumiendo que estos pueden distribuir su tiempo entre la docencia en la universidad pública, que es de carácter obligatorio, la investigación, y otra actividad alternativa, como por ejemplo la catedra en la universidad privada o la consultoría, con lo cual se incorpora el costo de oportunidad del tiempo dedicado a la investigación. El modelo es una adaptación en tiempo discreto del modelo de Levin y Stephan (1991). La modificación tiene que ver con el hecho de que la recompensa futura no depende del prestigio del investigador, sino que su expectativa de salario futuro depende de la productividad intelectual acumulada durante todo su ciclo vida académico.

Como en Levin y Stephan (1991), se asume que la función de utilidad de los profesores de la universidad pública en Colombia tiene como argumentos el status del profesor en la comunidad universitaria Status $_{t}$ y los bienes de consumo $X_{t}$.

$$
U_{t}=\ln \text { Status }_{t}^{\Theta_{1}}+\ln X_{t}^{\Theta_{2}}
$$

La variable Status $_{t}$ se calcula como una suma descontada de las publicaciones $P^{2}$ presentes y pasadas:

$$
\text { Status }_{t}=\sum_{s=0}^{t-1} \rho_{s t}^{s} * P_{t-s}
$$

El profesor maximiza el valor presente de su utilidad durante un periodo de tiempo T:

$$
V=\sum_{t=1}^{T} \rho^{t} * \ln \text { status }_{t}^{\Theta_{1}}+\ln X_{t}^{\Theta_{2}}
$$

Sujeto a:

$$
\begin{gathered}
\Delta k_{t}=\alpha P_{t-1}+\delta k_{t-1} \text { (4) } \\
\Delta A_{t}=r A_{t-1}-p X_{t}+W_{t}+\left(1-s_{t}\right) O W \text { (5) } \\
W_{t}=W_{0}+V P S * \sum_{s=1}^{t-1} P_{s} \text { (6) }
\end{gathered}
$$

2 En la simulación se asume que cada publicación corresponde a un punto salarial, en la realidad cada publicación es valorada según el artículo 10 del Decreto 1279. 
Donde $\rho$ y $\rho_{\text {st }}$ son parámetros de preferencia temporal de la función de utilidad y el status del profesor y $r$ es la tasa de interés. La ecuación (4) corresponde a la dinámica del stock de conocimiento $K_{t}$, que se actualiza en función de las publicaciones del periodo anterior $P_{t-1}$ y considerando una cierta tasa de depreciación del conocimiento $\delta$. La ecuación (5) describe la dinámica de los activos $A_{t}$, donde $p$ es el precio de $X$. La variable $s_{t}$ es la proporción de tiempo invertido en investigación, y (1 $-s_{t}$ ) la fracción de dedicada a trabajar en otra universidad o en consultoría, por la que recibe un pago $0 \mathrm{~W}$, que se asume exógeno a la productividad investigativa del docente. El término $W_{t}$ es el salario del periodo, que es función de la producción intelectual acumulada hasta el periodo anterior. La variable VPS es el valor del punto salarial.

La función de producción de investigación se especifica como:

$$
P_{t}=\left(s_{t} K_{t}\right)^{\beta}(7)
$$

Las variables de elección son $s_{t}$ y $X_{t}$. Se asumen $A_{t}=0$ y $K_{T}$ libre. La Tabla 1 contiene los parámetros iniciales para la simulación del modelo. En la Tabla 1, la relación entre los valores de los parámetros de la función de utilidad es $\Theta_{2}>\Theta_{1}$, para darle mayor peso al consumo de bienes que al status del docente en la universidad, y sea posible que el profesor dedique tiempo a otras actividades generadoras de ingreso. E1 parámetro $\beta<1$ para que la función de producción de investigación presente rendimientos marginales decrecientes en el stock de conocimiento $K$. Los valores de las tasas de descuento, de actualización del conocimiento $\mathrm{y}$ del valor del punto salarial, fueron asignados para que la simulación tuviera sentido, esto implica que la relación entre el salario inicial y el salario final sea conforme a la realidad, y que el profesor dedique alguna fracción de su tiempo disponible a otras actividades generadoras de ingreso. La simulación comprende 35 años, para un intervalo de edades del profesor representativo de los 31 a 65 años.

La Tabla 2 muestra la primera simulación del modelo. Se observa que hasta la edad de 51 años, el profesor representativo del modelo dedica cada vez más una fracción $(s)$ de su tiempo a la investigación, y menos a otras actividades que generan ingreso, como dar clases en otra universidad. Sin embargo, con el paso de los años, el valor presente del incentivo por publicar cae, lo que hace que ahora el profesor decida hacia el final de su carrera, comenzar a dedicar más proporción de su tiempo $(1-s)$ a otras actividades. La producción total del investigador durante su ciclo de vida es de $\Sigma$ vida $P_{t}=87,6$ puntos, la fracción de tiempo promedio dedicado a la investigación es $S_{1}=0,591$ y el salario promedio final es $W_{f}=11.617,5$, menos de cuatro veces el salario inicial. 
Salarios, incentivos y producción intelectual docente en la universidad pública ... Jhon Alexánder Méndez Sayago - Leonardo Vera Azaf

Tabla 1. Valores de los parámetros para la simulación del modelo en la línea base

\begin{tabular}{|c|c|}
\hline Parámetros & Valor \\
\hline \multicolumn{3}{|c|}{ Función de utilidad } \\
\hline$\Theta_{1}$ & 0,2 \\
\hline$\Theta_{2}$ & 0,8 \\
\hline \multicolumn{3}{|c|}{ Función de producción } \\
\hline \multicolumn{3}{|c|}{ Actualización conocimiento } \\
\hline$\alpha$ & 0,8 \\
\hline$\delta$ & 0,2 \\
\hline \multicolumn{3}{|c|}{ Tasas descuento } \\
\hline$\rho_{s t}$ & 0,1 \\
\hline$r$ & 0,04 \\
\hline $\mathbf{W}_{0}$ & 0,5 \\
\hline $0 W$ & 0,1 \\
\hline$V P S$ & $\mathbf{O t r o s}$ \\
\hline $\mathbf{K}_{0}$ & 10000 \\
\hline
\end{tabular}

Fuente: elaboración propia 
Apuntes Cenes Vol. 34, No. 60, ISSN 0120-3053 julio - diciembre 2015, Págs. 95-130

Tabla 2. Simulación del modelo - línea base

\begin{tabular}{|c|c|c|c|c|c|c|c|c|c|c|c|}
\hline Edad & Status & $\mathbf{X}$ & Utilidad & $\rho^{t}$ & $U^{d}$ & $s$ & A & $\mathbf{K}$ & $\mathbf{P}$ & W & OW \\
\hline 31 & 1,46 & 6,22 & 2,44 & 1,00 & 2,444 & 0,80 & 0,0 & 2 & 1,46 & 3000,0 & 1978,7 \\
\hline 32 & 1,85 & 6,54 & 2,56 & 0,96 & 2,464 & 0,51 & 2781,9 & 2,238 & 1,12 & 3146,0 & 4871,4 \\
\hline 33 & 2,03 & 6,88 & 2,62 & 0,92 & 2,427 & 0,48 & 5993,4 & 2,349 & 1,10 & 3257,6 & 5183,2 \\
\hline 34 & 2,16 & 7,24 & 2,68 & 0,89 & 2,379 & 0,48 & 9330,8 & 2,445 & 1,14 & 3368,0 & 5165,0 \\
\hline 35 & 2,28 & 7,62 & 2,72 & 0,85 & 2,329 & 0,49 & 12716,2 & 2,544 & 1,20 & 3482,3 & 5068,1 \\
\hline 36 & 2,40 & 8,02 & 2,77 & 0,82 & 2,279 & 0,51 & 16109,2 & 2,649 & 1,26 & 3602,2 & 4937,0 \\
\hline 37 & 2,54 & 8,44 & 2,82 & 0,79 & 2,228 & 0,52 & 19483,4 & 2,764 & 1,34 & 3728,7 & 4789,7 \\
\hline 38 & 2,69 & 8,89 & 2,87 & 0,76 & 2,178 & 0,54 & 22819,7 & 2,889 & 1,42 & 3862,6 & 4636,2 \\
\hline 39 & 2,85 & 9,36 & 2,91 & 0,73 & 2,129 & 0,55 & 26095,6 & 3,026 & 1,51 & 4004,5 & 4475,4 \\
\hline 40 & 3,03 & 9,85 & 2,96 & 0,70 & 2,079 & 0,57 & 29283,5 & 3,176 & 1,61 & 4155,4 & 4304,8 \\
\hline 41 & 3,23 & 10,37 & 3,01 & 0,68 & 2,031 & 0,59 & 32356,1 & 3,340 & 1,71 & 4316,0 & 4127,6 \\
\hline 42 & 3,45 & 10,92 & 3,05 & 0,65 & 1,983 & 0,61 & 35287,9 & 3,520 & 1,83 & 4487,4 & 3948,7 \\
\hline 43 & 3,68 & 11,51 & 3,10 & 0,62 & 1,935 & 0,62 & 38054,0 & 3,718 & 1,96 & 4670,6 & 3771,2 \\
\hline 44 & 3,93 & 12,12 & 3,14 & 0,60 & 1,887 & 0,64 & 40627,8 & 3,933 & 2,09 & 4866,3 & 3596,6 \\
\hline 45 & 4,21 & 12,76 & 3,19 & 0,58 & 1,841 & 0,66 & 42982,1 & 4,168 & 2,24 & 5075,7 & 3427,4 \\
\hline 46 & 4,50 & 13,45 & 3,23 & 0,56 & 1,795 & 0,67 & 45091,1 & 4,423 & 2,39 & 5299,6 & 3268,2 \\
\hline 47 & 4,80 & 14,17 & 3,28 & 0,53 & 1,749 & 0,69 & 46931,3 & 4,699 & 2,56 & 5539,0 & 3124,5 \\
\hline 48 & 5,12 & 14,92 & 3,32 & 0,51 & 1,704 & 0,70 & 48481,5 & 4,996 & 2,72 & 5794,6 & 3002,1 \\
\hline 49 & 5,45 & 15,73 & 3,36 & 0,49 & & 0,71 & & 5,313 & 2,89 & 6066,7 & 2905,8 \\
\hline 50 & 5,78 & 16,57 & 3,40 & 0,47 & 1,615 & 0,72 & 50634,0 & 5,648 & 3,06 & 6355,8 & 2839,9 \\
\hline 51 & 6,11 & 17,46 & 3,44 & 0,46 & 1,572 & 0,72 & 51198,9 & 6,001 & 3,22 & 6661,6 & 2808,7 \\
\hline 52 & 6,43 & 18,40 & 3,48 & 0,44 & 1,529 & 0,72 & 51397,9 & 6,367 & 3,37 & 6983,7 & 2816,6 \\
\hline 53 & 6,73 & 19,39 & 3,52 & 0,42 & 1,487 & 0,71 & 51211,3 & 6,743 & 3,51 & 7321,2 & 2867,5 \\
\hline 54 & 6,99 & 20,44 & 3,56 & 0,41 & 1,445 & 0,70 & 50616,0 & 7,123 & 3,63 & 7672,5 & 2963,8 \\
\hline 55 & 7,22 & 21,55 & 3,60 & 0,39 & 1,404 & 0,69 & 49582,9 & 7,500 & 3,72 & 8035,6 & 3106,1 \\
\hline 56 & 7,39 & 22,71 & 3,64 & 0,38 & 1,364 & 0,67 & 48072,1 & 7,866 & 3,78 & 8407,8 & 3292,0 \\
\hline 57 & 7,51 & 23,94 & 3,67 & 0,36 & 1,324 & 0,65 & 46029,8 & 8,215 & 3,81 & 8786,1 & 3517,0 \\
\hline 58 & 7,56 & 25,24 & 3,71 & 0,35 & 1,285 & 0,62 & 43383,6 & 8,537 & 3,81 & 9167,3 & 3774,1 \\
\hline 59 & 7,55 & 26,61 & 3,74 & 0,33 & 1,247 & 0,59 & 40038,9 & 8,825 & 3,77 & 9547,8 & 4055,0 \\
\hline 60 & 7,47 & 28,05 & 3,77 & 0,32 & 1,209 & 0,56 & 35876,2 & 9,072 & 3,70 & 9924,4 & 4350,7 \\
\hline 61 & 7,33 & 29,58 & 3,80 & 0,31 & 1,172 & 0,53 & 30750,8 & 9,274 & 3,60 & 10294,0 & 4654,3 \\
\hline 62 & 7,14 & 31,19 & 3,83 & 0,30 & 1,136 & 0,50 & 24505,3 & 9,426 & 3,47 & 10653,9 & 4974,1 \\
\hline 63 & 6,84 & 32,88 & 3,86 & 0,29 & 1,100 & 0,46 & 17037,6 & 9,525 & 3,27 & 11001,0 & 5387,6 \\
\hline 64 & 6,20 & 34,67 & 3,88 & 0,27 & 1,064 & 0,38 & 8563,2 & 9,552 & 2,79 & 11327,8 & 6233,3 \\
\hline 65 & 4,60 & 36,59 & 3,87 & 0,26 & 1,021 & 0,18 & 0,0 & 9,433 & 1,50 & 11606,3 & 8244,2 \\
\hline
\end{tabular}

Fuente: elaboración propia. 
La Figura 1 muestra las dinámicas del stock de conocimiento y el número de publicaciones durante el ciclo de vida del profesor investigador. Se observa que a pesar de que el profesor acumula conocimiento durante casi toda su vida como docente universitario, su producción académica declina desde los cincuenta y ocho años, esto debido a que como se observa en la trayectoria $s_{1}$ de la
Figura 2, la fracción de tiempo dedicada a la investigación cae a partir de los 51 años, como consecuencia de la reducción en el valor presente del incentivo a la investigación. También se puede notar que la relación entre la edad del profesor y sus publicaciones es convexa en los primeros años y después se convierte en cóncava.

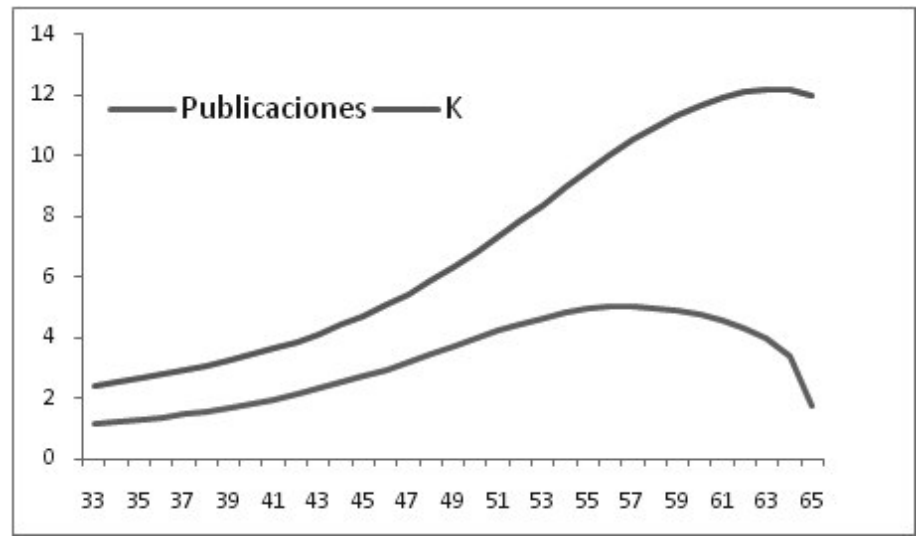

Figura 1. Dinámicas del stock de conocimiento y de las publicaciones.

Fuente: elaboración propia

Si el salario de enganche aumenta a $W_{0}$ $=400$, la producción total del investigador durante su ciclo de vida aumenta a ¿vida $P_{t}=108,9$ puntos, debido a que aumenta la fracción de tiempo promedio dedicado a la investigación, que ahora toma el valor de $S_{2}=0,653$. El salario promedio final es de $W_{f}=14.720,5$. Es decir, este cambio exógeno en el salario permite observar el efecto directo del salario sobre la producción intelectual.

Si sumado al incremento en el salario inicial, también aumenta el valor del punto salarial, y VPS pasa de 100 a 110 , la producción total del investigador durante su ciclo de vida aumenta a Svida $P_{t}=173,05$ puntos, que resulta del aumento en la fracción de tiempo promedio dedicado a la investigación $S_{3}=0,792$. Se concluye entonces que el salario y el valor del punto salarial tienen efecto positivo sobre la productividad intelectual del profesor representativo del modelo.

La Figura 2 muestra las gráficas de las fracciones de tiempo dedicadas en la 
investigación en la línea base, y cuando se presentan los cambios exógenos en el salario y el valor del punto salarial. Se observa que al aumentar el salario y el valor del punto salarial exógenamente, la fracción de tiempo dedicada a la investigación es más alta para cada año.
En los dos primeros casos, el pico más alto de la fracción de tiempo dedicado a la investigación se alcanza a los 51 años. Cuando se aumenta el valor del punto salarial el profesor dedica todo su tiempo disponible para la investigación hasta los 55 años.

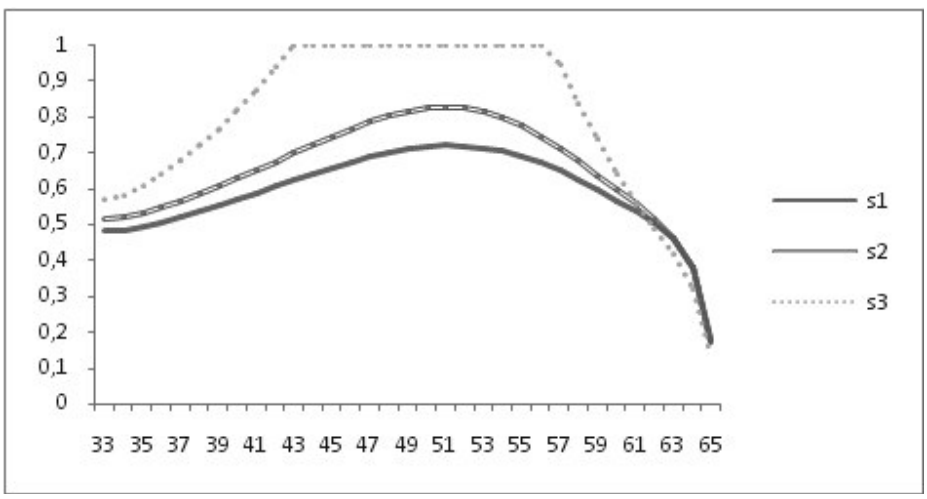

Figura 2. Fracción de tiempo dedicada a la investigación en cada simulación

Fuente: elaboración propia

\section{DATOS}

Esta sección tiene como propósito realizar una rápida descripción de la información disponible para la estimación del modelo econométrico de la productividad intelectual de los profesores universitarios. Los datos corresponden a información del cuerpo de profesores de la Universidad del Valle, para el periodo comprendido entre los años 2004-2012. La información comprende: fecha de ingreso a la universidad (año de contratación), edad, salario, facultad a la que pertenece, nivel académico, categoría, pertenencia a un grupo de investigación, puntos por producción intelec- tual, puntaje por docencia destacada y puntos por dirección académico administrativa de los profesores nombrados de la Universidad del Valle, sometidos al Decreto 1279, por el cual se establece el régimen salarial y prestacional de los docentes de las universidades estatales en Colombia.

La Tabla 3 muestra el promedio por facultad de los puntos por productividad académica resultado de la actualización salarial anual y su desviación estándar. El promedio más alto corresponde a los profesores de la facultad de ciencias, y el promedio más bajo es el de los profesores de la facultad de psicología. 
Salarios, incentivos y producción intelectual docente en la universidad pública ... Jhon Alexánder Méndez Sayago - Leonardo Vera Azaf

Tabla 3. Promedio anual de los puntos por productividad académica por facultad

\begin{tabular}{|c|c|c|c|c|}
\hline Facultad & Obs. & Prom. Anual & Desv. Est. & $\begin{array}{c}\text { Representación } \\
\text { Var. Binaria } \boldsymbol{d}=\mathbf{1}\end{array}$ \\
\hline Artes & 806 & 3,09 & 9,31 & dartes \\
Administración & 302 & 4,23 & 13,04 & dadmon \\
Ingeniería & 1053 & 6,64 & 16,24 & dingenieria \\
Socio economía & 176 & 5,65 & 12,29 & dsocioeconomía \\
Ciencias & 753 & 10,18 & 21,85 & dciencias \\
Psicología & 145 & 2,91 & 8,63 & dpisicologia \\
Salud & 1158 & 3,44 & 11,22 & dsalud \\
Humanidades & 781 & 3,88 & 8,54 & dhumanidades \\
\hline
\end{tabular}

Fuente: elaboración propia.

La Tabla 4 presenta el promedio de los puntos por productividad académica por nivel académico y su desviación estándar. Los profesores con doctorado presentan la productividad más alta, seguida por la productividad de los profesores con maestría como era de esperarse, siendo la de los primeros más de dos veces y medio más alta que la de los segundos. Los profesores con especialización no parecen tener ventaja investigativa sobre los profesores con pregrado, porque su productividad es inferior.

La Tabla 5 presenta el promedio de los puntos por productividad académica según la categoría del profesor y su desviación estándar. Se observa que el promedio de puntos por productividad académica de los profesores, aumenta con el grado académico alcanzado.

Tabla 4. Promedio anual de los puntos por productividad académica por nivel académico

\begin{tabular}{|c|c|c|c|c|}
\hline $\begin{array}{c}\text { Nivel } \\
\text { académico }\end{array}$ & Obs. & Prom. Anual & Desv. Est. & $\begin{array}{c}\text { Representación } \\
\text { Var. Binaria } \boldsymbol{d}=\mathbf{1}\end{array}$ \\
\hline Pregrado & 650 & 2,89 & 9,60 & dpregrado \\
Especialización & 925 & 1,98 & 6,86 & despecializacion \\
Maestría & 2101 & 3,81 & 10,27 & dmaestria \\
Doctorado & 1498 & 10,10 & 20,83 & ddoctorado \\
\hline
\end{tabular}

Fuente: elaboración propia.

Tabla 5. Promedio anual de los puntos por productividad intelectual según categoría

\begin{tabular}{|c|c|c|c|c|}
\hline Categoría & Obs. & Prom. Anual & Desv. Est. & $\begin{array}{c}\text { Representación } \\
\text { Var. Binaria } \boldsymbol{d}=\mathbf{1}\end{array}$ \\
\hline Auxiliar & 921 & 2,20 & 5,85 & dauxiliar \\
Asistente & 1629 & 4,21 & 12,00 & dasistente \\
Asociado & 909 & 5,65 & 13,09 & dasociado \\
Titular & 1715 & 7,49 & 18,50 & dtitular \\
\hline
\end{tabular}

Fuente: elaboración propia. 
La Tabla 6 exhibe las estadísticas descriptivas del promedio durante los años 2004-2011 de la edad de los profesores, el salario real a precios del año 2007 y los puntos acumulados por docencia destacada y productividad académica.

Tabla 6. Estadísticas descriptivas de las variables continuas

\begin{tabular}{|c|c|c|c|c|c|c|}
\hline Variable & Obs. & Prom. Anual & Desv. Est. & Min. & Max. & $\begin{array}{c}\text { Representación } \\
\text { Var. Binaria } \boldsymbol{d}=\mathbf{1}\end{array}$ \\
\hline Edad & 6059 & 48,7 & 8,6 & 25 & 84 & Edad \\
\hline Salario real & 6059 & $3^{\prime} 526.821$ & $1^{\prime} 763.153$ & 824.942 & $15^{\prime} 100.000$ & Salario \\
\hline $\begin{array}{c}\text { Puntos por } \\
\text { docencia } \\
\text { destacada }\end{array}$ & 6059 & 7,9 & 8,7 & 0,0 & 50,0 & puntos_dd \\
\hline $\begin{array}{c}\text { Puntos por } \\
\text { productividad } \\
\text { intelectual }\end{array}$ & 6059 & 91,5 & 135,9 & 0,0 & 1350,9 & $\mathrm{P}$ \\
\hline
\end{tabular}

Fuente: elaboración propia.

ESTIMACIÓN DEL MODELO La especificación del modelo economéECONOMÉTRICO Y ANÁLISIS DE trico para la producción intelectual doLOS RESULTADOS cente es la siguiente:

$P_{i t}=\beta_{0}+\gamma *$ incentivo $_{t}+\delta_{1}$ salario $_{\mathrm{i}, \mathrm{t}-1}+\delta_{2}$ salario $_{\mathrm{i}, \mathrm{t}-1}^{2}+\sum_{i=1}^{k} B j * X_{i t j}+a_{i}+u_{i t}$

Donde:

$P_{i t}:$ puntos por producción intelectual del profesor $i$ en el periodo $t$.

incentivo $_{t}$ : equivale al valor presente neto de las ganancias esperadas de cada punto de productividad intelectual en el periodo $t$.

salario $_{i, t-1}$ : salario real del docente $i$ en el periodo $t-1$, periodo en el cual el profesor inicia la investigación que conduce a la publicación en el periodo siguiente.
El vector $X$ contiene información sobre otras variables que pueden incidir en la productividad intelectual como máximo grado académico del profesor, área de la ciencia a la que pertenece (Facultad o instituto), pertenencia a un grupo de investigación, antigüedad, puntos por producción intelectual, categoría etc.

La expresión para el cálculo de la variable incentivo $_{t}$ es la siguiente:

$$
\text { incentivo }_{t}=V P S * N P A * \frac{\mid 1-(1+\delta)^{-\left(\text {edad de jubilación - edad actual }{ }_{t}\right)}}{\delta}
$$


En (9), el término VPS corresponde al valor del punto salarial, NPA es el número de pagos al año que recibe el profesor, y $\delta$ es la tasa de descuento que se establece exógenamente. Los valores asignados son: $V P S=10.745$ y $N P A=$ 15.

La especificación del modelo (8) propone una relación entre salarios reales y productividad intelectual que tiene forma de $\mathrm{U}$ invertida y sigue a Tang (2010). La razón que expone el autor es la siguiente: en principio, el aumento inicial de los salarios a los empleados logra que estos sean más productivos, porque el aumento de los salarios hace el ocio más caro. No obstante, el incremento adicional de los salarios disminuirá la productividad, porque los empleados se hacen más ricos y pueden permitirse más tiempo libre.
Para tener una primera aproximación de los factores que determinan la publicación de los profesores, se estima un modelo Logit donde la variable dependiente es la variable binaria publicacion. La variable toma el valor de uno si el profesor realizó actualización salarial en el periodo ${ }^{3}$, por lo cual recibió puntos por productividad intelectual, y cero en el otro caso contrario.

La estimación del modelo Logit aparece en la Tabla 7. La edad, el salario, los puntos por productividad intelectual y docencia destacada están rezagados, para tener en cuenta el momento en el que se tomó la decisión de investigar para realizar la publicación del artículo o libro de interés. Las variables de categoría del profesor, el salario real rezagado en niveles, y las variables binarias de las facultades no resultan significativas, excepto las dummy de humanidades y psicología.

Cada periodo corresponde a dos años, asumiendo ciclos de investigación-publicación cada par de años. 
Tabla 7. Modelo Logit de efectos aleatorios para establecer los determinantes de la publicación

\begin{tabular}{|c|c|c|c|c|}
\hline Var. Dep. Binaria & $p u b$ & acion & & \\
\hline Observaciones & \multicolumn{3}{|c|}{2509} & \\
\hline Número de grupos & \multicolumn{2}{|c|}{771} & & \\
\hline Var. Explicativa & Coeficiente & Error estándar & $\mathbf{z}$ & $P>\mathbf{Z}$ \\
\hline dasistente & 0.1492 & 0.1888 & 0.79 & 0.430 \\
\hline dasociado & 0,4376 & 0.2570 & 1.70 & 0.089 \\
\hline dtitular & -0.0582 & 0.3229 & -0.18 & 0.857 \\
\hline despecializacion & 0.4956 & 0.2340 & 2.12 & 0.034 \\
\hline dmaestria & 0.6836 & 0.2122 & 3.24 & 0.001 \\
\hline ddoctorado & 1.3992 & 0.2467 & 5.67 & 0.000 \\
\hline$\in$ grupo de investigacion & 0.8850 & 0.1417 & 6.24 & 0.000 \\
\hline$e d a d_{\mathrm{t}-1}$ & -0.050 & 0.0095 & -5.27 & 0.000 \\
\hline salario $_{\mathrm{t}-1}$ & $-5.07 e-09$ & $1.67 \mathrm{e}-07$ & -0.03 & 0.976 \\
\hline salario $_{\mathrm{t}-1}$ & $-6.63 e-14$ & $1.82 \mathrm{e}-14$ & -3.64 & 0.000 \\
\hline puntos_pa $a_{\mathrm{t}-1}$ & 0.0126 & 0.0016 & 7.85 & 0.000 \\
\hline puntos_dd ${ }_{\mathrm{t}-1}$ & 0.0216 & 0.0088 & 2.45 & 0.014 \\
\hline dhumanidades & 0.7764 & 0.2162 & 3.59 & 0.000 \\
\hline dpsicologia & -0.7529 & 0.4008 & -1.88 & 0.060 \\
\hline dartes & -0.0140 & 0.2114 & -0.07 & 0.947 \\
\hline dsocioeconomia & 0.2115 & 0.3480 & 061 & 0.543 \\
\hline dadministracion & -0.1143 & 0.2930 & -0.39 & 0.696 \\
\hline dingenieria & 0.3244 & 0.2113 & 1.53 & 0.125 \\
\hline dciencias & 0.0287 & 0.2476 & 0.12 & 0.908 \\
\hline$d 2008$ & 0.3023 & 0.1503 & 2.01 & 0.044 \\
\hline$d 2010$ & 0.2590 & 0.1562 & 1.66 & 0.097 \\
\hline$d 2012$ & 0.5817 & 0.1710 & 3.40 & 0.001 \\
\hline
\end{tabular}

Fuente: elaboración propia

La Tabla 8 presenta en las columnas (II) y (III) la estimación de los modelos de ciclo de vida y producción intelectual, mediante la estimación Tobit de efectos aleatorios. La única diferencia entre los modelos de ciclo de vida y de producción intelectual, es que el primero utiliza la edad como regresor, y en el segundo la variable explicativa de interés es el incentivo por producción intelectual.
Por otra parte, puede existir sesgo en los estimadores del modelo Tobit de efectos aleatorios si la heterogeneidad individual no observada, tiene la forma de efectos fijos correlacionados con las variables explicativas. Para superar este problema, Levin y Stephan (1991) estiman un modelo Tobit que incluye dummys individuales, Goodwin $\mathrm{y}$ Sauer (1995) y Rauber y Ursprung 
(2008) incorporan una variable categórica resultado de ranquear a los investigadores en quintiles a partir de la producción promedio de su ciclo de vida. Siguiendo una estrategia semejante, Vella y Verbeck (1997) utilizan los residuales de una regresión preliminar para ranquear a los investigadores en función de su habilidad investigativa no capturada por la parte determinística del modelo. En las columnas (IV) y (V) aparecen las estimaciones de los modelos de ciclo de vida y de producción intelectual utilizando la metodología de Vella y Verbeck (1997) para capturar la heterogeneidad individual fija.

Las tasas de descuento utilizadas en los modelos de producción intelectual fueron: $\delta=0,000$ (no hay tasa de descuento) en el modelo Tobit de efectos aleatorios y $\delta=0,019$ para el modelo Tobit que sigue la metodología de Vella y Verbeck (1997). Las tasas fueron escogidas porque maximizaban la función de verosimilitud.

Como era de esperarse, a medida que aumenta el nivel académico del profesor, mejora su producción intelectual. Las estimaciones muestran que un profesor con doctorado tiene aproximadamente 6 puntos más de producción intelectual que un profesor con maestría, y casi ocho puntos más que un profesor con especialización.

Pertenecer a un grupo de investigación tiene efecto positivo sobre la producción intelectual de aproximadamente seis puntos de producción intelectual al año.

El efecto de la edad es negativo, porque a medida que el profesor envejece, la ganancia esperada de las publicaciones cae. Si bien con el paso de los años el profesor investigador mejora su stock de conocimiento, y por tanto sus habilidades para publicar, este efecto positivo es captado por las variables productividad intelectual acumulada rezagada (puntos_pa_l) y los puntos por docencia destacada acumulada rezagada (puntos_dd_l), así que no se requiere forma cuadrática para la edad.

El efecto del incentivo es positivo y significativo. La idea al incluir las variables puntos_pa_1 y puntos_dd_1 es que el efecto del incentivo no incluya otros efectos asociados con la edad. De hecho si no se incluyen este par de variables, la edad y la edad al cuadrado resultan significativas, con sus respectivos signos positivo y negativo. Por tanto, si no se incluyera como regresores las variables puntos_pa_1 y puntos_dd_1, el efecto del incentivo sería subestimado.

Se debe destacar también que a pesar de la incorporación de la producción media del profesor durante su ciclo de vida (residual) como variable explicativa en el modelo Tobit, la variable (puntos $p a_{t-1}$ ) sigue siendo significativa, lo que demuestra que los aportes individuales de estas dos variables al modelo son independientes. $\mathrm{La}$ variable residual también es significativa. 
La Facultad de Ciencias presenta ceteris paribus la mayor producción intelectual, que estaría casi 3 puntos por encima del promedio de la Universidad. En la estimación Tobit de efectos aleatorios, la menor producción intelectual ceteris paribus es la del Instituto de Psicología. En la estimación Tobit de Vella y Verbeck (1997), la Facultad de Ciencias presenta ceteris paribus la menor producción intelectual.

La variable salario real rezagada en niveles no es significativa en ninguna estimación, y la misma variable elevada al cuadrado resultó significativa (solo en la estimación Tobit de efectos aleatorios) y su efecto negativo, porque a niveles de salarios muy altos el profesor prefiere el ocio al ingreso o al status en la universidad.

Las variables binarias de periodo se incluyen para capturar el efecto de cohorte siguiendo la recomendación de Rauber y Ursprung (2005). Las dummy de tiempo revelan el rápido crecimiento de la producción intelectual de los profesores de la Universidad del Valle, no asociada con las características individuales observadas.
Los efectos estimados del incentivo para la producción intelectual fueron $\gamma_{t E A}=3.16 * 10^{-6}$ para el modelo Tobit de efectos aleatorios y $\gamma_{t V V}=3.44 *$ $10^{-6}$ en el modelo Tobit que sigue la metodología de Vella y Verbeck (1997). Este último es aproximadamente un 8,8 $\%$ más alto que el del modelo Tobit con efectos aleatorios, pero hay que tener en cuenta que en la estimación Tobit de efectos aleatorios al incentivo no se le aplica tasa de descuento.

Las figuras 3 y 4 muestran el efecto estimado del incentivo ceteris paribus, sobre la productividad intelectual de los profesores de la Universidad del Valle, calculados a partir del modelo Tobit de efectos aleatorios (Figura 3) y el modelo Tobit de Vella y Verbeck (Figura 4). En el primero, el efecto es de 20,3 puntos para un profesor de 30 años, de 15,27 puntos para un profesor de 40 años, de 10,18 puntos para un profesor de 50 años, y de 5 puntos para un profesor de 60 años. En el segundo el efecto es de 15,4 puntos para un profesor de 30 años, de 12,59 puntos para un profesor de 40 años, de 9,15 puntos para un profesor de 50 años, y de casi 5 puntos para un profesor de 60 años. 
Salarios, incentivos y producción intelectual docente en la universidad pública ... Jhon Alexánder Méndez Sayago - Leonardo Vera Azaf

Tabla 8. Estimación de modelos de ciclo de vida y de producción intelectual

\begin{tabular}{|c|c|c|c|c|}
\hline \multirow{3}{*}{\begin{tabular}{|c|} 
Variable dependiente \\
Número de observaciones \\
\end{tabular}} & \multicolumn{4}{|c|}{ Puntos por producción intelectual $(P)$} \\
\hline & \multicolumn{4}{|c|}{2509} \\
\hline & \multicolumn{2}{|c|}{$\begin{array}{c}\text { Estimación Tobit } \\
\text { de efectos aleatorios }\end{array}$} & \multicolumn{2}{|c|}{$\begin{array}{l}\text { Estimación Tobit - metodología } \\
\text { de Vella y Verbeck (1997) }\end{array}$} \\
\hline Var. Explicativa & $\begin{array}{l}\text { Modelo de } \\
\text { ciclo de } \\
\text { vida(II) }\end{array}$ & $\begin{array}{l}\text { Producción } \\
\text { intelectual } \\
\text { (III) }\end{array}$ & $\begin{array}{l}\text { Modelo de } \\
\text { ciclo de } \\
\text { vida (IV) }\end{array}$ & $\begin{array}{l}\text { Producció } \\
\text { intelectual } \\
\text { (V) }\end{array}$ \\
\hline despecializacion & $\begin{array}{c}3.1452 \\
0.077\end{array}$ & $\begin{array}{c}3.1230 \\
0.088\end{array}$ & $\begin{array}{c}3.0889 \\
0.049\end{array}$ & $\begin{array}{c}3.0739 \\
0.050\end{array}$ \\
\hline dmaestria & $\begin{array}{c}6.1391 \\
0.000\end{array}$ & $\begin{array}{c}6.000 \\
0.000\end{array}$ & $\begin{array}{c}4.9007 \\
0.000\end{array}$ & $\begin{array}{c}4.0032 \\
0.000\end{array}$ \\
\hline ddoctorado & $\begin{array}{c}12.6735 \\
0.000\end{array}$ & $\begin{array}{c}12.6659 \\
0.000\end{array}$ & $\begin{array}{c}10.9312 \\
0.000\end{array}$ & $\begin{array}{r}10.9147 \\
0.000\end{array}$ \\
\hline$\in$ grupo de investigacion & $\begin{array}{c}6.1416 \\
0.000\end{array}$ & $\begin{array}{c}6.1376 \\
0.000\end{array}$ & $\begin{array}{c}5.8459 \\
0.000\end{array}$ & $\begin{array}{c}5.8402 \\
0.000\end{array}$ \\
\hline edad & $\begin{array}{l}-0.5108 \\
0.000\end{array}$ & - & $\begin{array}{c}-0.5333 \\
0.000\end{array}$ & - \\
\hline Incentivo $_{\mathrm{t}}$ & - & $\begin{array}{c}3.16 \mathrm{e}-6 \\
0.000\end{array}$ & - & $\begin{array}{c}3.44 \mathrm{e}-6 \\
0.000\end{array}$ \\
\hline Salario $_{\mathrm{t}-1}$ & $\begin{array}{c}-6.03 e-13 \\
0.000\end{array}$ & $\begin{array}{c}-6.04 \mathrm{e}-13 \\
0.000\end{array}$ & - & - \\
\hline puntos_pa $a_{\mathrm{t}-1}$ & $\begin{array}{c}0.1285 \\
0.000\end{array}$ & $\begin{array}{c}0.1286 \\
0.000\end{array}$ & $\begin{array}{c}0.0576 \\
0.000\end{array}$ & $\begin{array}{c}0.0576 \\
0.000\end{array}$ \\
\hline puntos_dd $d_{\mathrm{t}-1}$ & $\begin{array}{c}0.1475 \\
0.019\end{array}$ & $\begin{array}{c}0.1475 \\
0.020\end{array}$ & $\begin{array}{c}0.0962 \\
0.071 \\
\end{array}$ & $\begin{array}{r}0.0959 \\
0.072 \\
\end{array}$ \\
\hline Residual & - & $\begin{array}{c}- \\
0.000 \\
\end{array}$ & $\begin{array}{c}1.1096 \\
0.000\end{array}$ & 1.1096 \\
\hline dhumanidades & $\begin{array}{l}3.261 \\
0.011 \\
\end{array}$ & $\begin{array}{c}3.2567 \\
0.012 \\
\end{array}$ & $\begin{array}{c}2.7849 \\
0.006 \\
\end{array}$ & $\begin{array}{c}2.7754 \\
0.007 \\
\end{array}$ \\
\hline dciencias & - & - & $\begin{array}{c}-2.5572 \\
0.027\end{array}$ & $\begin{array}{c}-2.5540 \\
0.027 \\
\end{array}$ \\
\hline dpsicologia & $\begin{array}{c}-5.6811 \\
0.060\end{array}$ & $\begin{array}{c}-5.6844 \\
0.060\end{array}$ & - & - \\
\hline$d 2008$ & $\begin{array}{c}2.9069 \\
0.013 \\
\end{array}$ & $\begin{array}{c}2.9276 \\
0.014 \\
\end{array}$ & $\begin{array}{c}2.0193 \\
0.072 \\
\end{array}$ & $\begin{array}{c}2.0251 \\
0.071\end{array}$ \\
\hline$d 2010$ & $\begin{array}{c}3.9471 \\
0.001 \\
\end{array}$ & $\begin{array}{c}3.9617 \\
0.001 \\
\end{array}$ & $\begin{array}{c}3.3067 \\
0.004 \\
\end{array}$ & $\begin{array}{c}3.3147 \\
0.003 \\
\end{array}$ \\
\hline$d 2012$ & $\begin{array}{c}8.1645 \\
0.000\end{array}$ & $\begin{array}{c}8.1932 \\
0.000\end{array}$ & $\begin{array}{c}6.3004 \\
0.000\end{array}$ & $\begin{array}{c}6.3135 \\
0.000\end{array}$ \\
\hline
\end{tabular}

Fuente: elaboración propia 


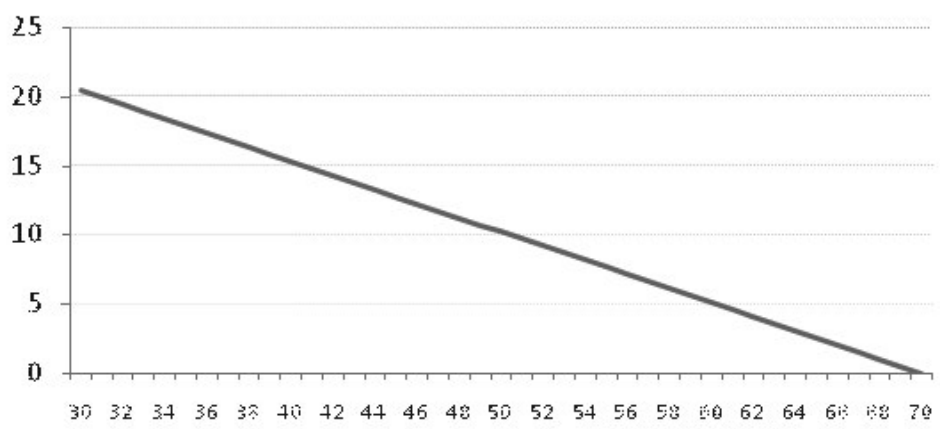

Figura 3. Efecto estimado del incentivo sobre la producción intelectual calculado a partir de la estimación Tobit de efectos aleatorios.

Fuente: elaboración propia

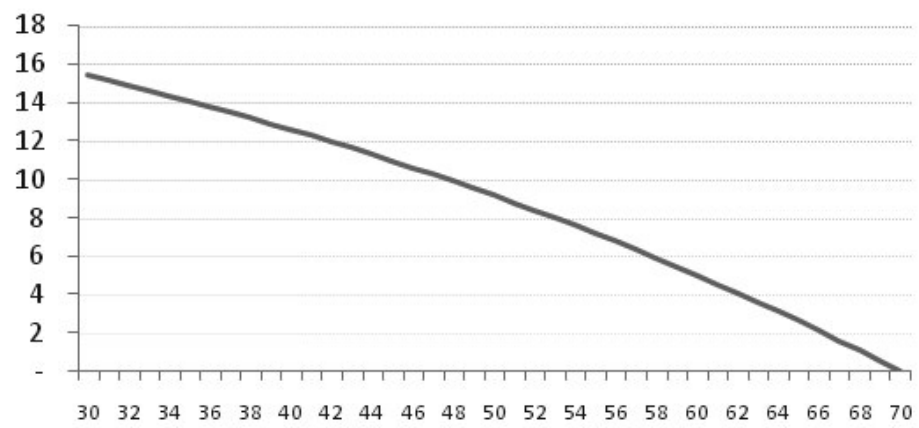

Figura 4. Efecto estimado del incentivo sobre la producción intelectual calculado a partir de la estimación Tobit de Vella y Verbeck (1997).

Fuente: elaboración propia

La Figura 5 revela los efectos estimados utilizando los resultados del modelo Tobit con efectos fijos, de incrementos del $10 \%, 20 \%, 30 \%, 40 \%$ y $50 \%$ en el valor del punto salarial sobre la producción intelectual, asociados a cada nivel de edad. 
Salarios, incentivos y producción intelectual docente en la universidad pública ... Jhon Alexánder Méndez Sayago - Leonardo Vera Azaf

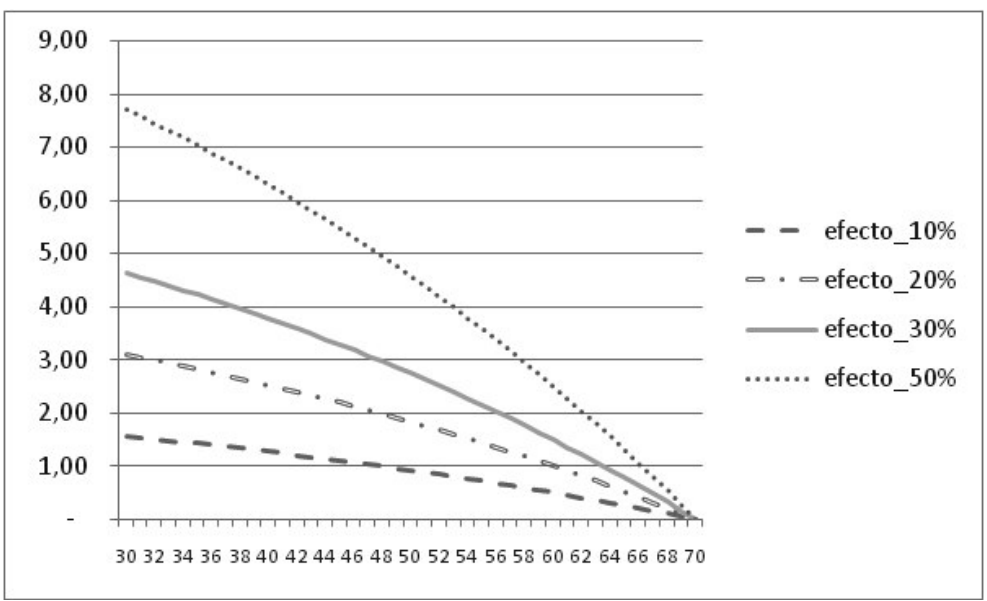

Figura 5. Efecto estimado del incremento porcentual del valor de punto salarial.

Fuente: elaboración propia

\section{CONCLUSIONES}

En el año 2012, de 759 profesores nombrados cobijados por el Decreto 1279, el $23.8 \%$ eran profesores sin maestría ni doctorado, el $44.3 \%$ profesores con maestría y el $31.8 \%$ profesores con doctorado. Las estimaciones encontraron que un profesor con doctorado tiene aproximadamente 6 puntos más de producción intelectual que un profesor con maestría, y casi ocho puntos más que un profesor con especialización. La primera diferencia es equivalente a la publicación de un par de artículos de categoría $\mathrm{C}$ al año y la segunda a un artículo de categoría $\mathrm{B}$ al año. Por tanto, existe la oportunidad de incrementar la producción intelectual, a través de la política de comisiones de estudio para mejorar el nivel académico de los profesores.
Se encontró que la participación en un grupo de investigación afecta la producción intelectual en casi seis puntos, un aporte similar a la diferencia en producción intelectual entre un profesor con doctorado y uno con maestría. Por tal razón, es importante que las autoridades universitarias fomenten la participación profesoral en los grupos de investigación.

En el modelo teórico desarrollado en este artículo, el salario del profesor afecta su producción intelectual, porque entre más alto sea su salario en la universidad pública, el profesor dedica menos tiempo a otras actividades generadoras de ingreso, como por ejemplo trabajar en otra universidad o en consultoría. Sin embargo, la evidencia empírica obtenida a partir de las estimaciones revela que el 
nivel de salario no tiene efecto positivo sobre la producción intelectual de los profesores de la universidad pública. Esto puede considerarse un indicio de que no existe tradeoff entre el tiempo dedicado a la investigación y el destinado a otras actividades generadoras de ingreso.

En el artículo de Levin y Stephan (1991), el salario del profesor resultó significativo en la explicación de su producción investigativa, pero hay que resaltar que en esa estimación se utilizó el salario como una proxy de las ganancias futuras de las publicaciones del docente. En este artículo se cuanta con una medición más aproximada de la utilidad marginal de la investigación (el valor presente del punto salarial).

Sin embargo, dado que la muestra de estimación abarca solo profesores de la Universidad del Valle, una universidad pública con salarios de enganche relativamente bajos, no se puede determinar el efecto del salario en la contratación de docentes con mejores habilidades investigativa, y por tanto, su efecto en la producción intelectual. Dada esta limitación, lo que se puede es que el nivel de salario no afecta la producción intelectual a través de la distribución del tiempo dedicado a la investigación.

Las estimaciones encontraron un efecto promedio del incentivo salarial sobre la productividad intelectual de 15,4 puntos para un profesor de 30 años, de 12,59 puntos para un profesor de 40 años, de 9,15 puntos para un profesor de 50 años, y de casi 5 puntos para un profesor de 60 años etc. Lo que revela claramente la importancia del factor incentivo, si lo comparamos con la diferencia estimada en el efecto sobre la producción intelectual, entre tener doctorado y maestría que es de seis puntos o menos, o la pertenencia a un grupo de investigación que es de seis puntos.

La mejor estimación en el modelo Tobit de Vella y Verbeck (1997) se obtuvo con una tasa de descuento del 1,9\%, y en la estimación Tobit de efectos aleatorios sin tasa de descuento. Dada la magnitud tan baja de la tasa de descuento, se puede concluir que la estrategia de algunas universidades privadas, de ofrecer primas por producción intelectual durante un periodo limitado (un año), no compite con el incentivo de la universidad pública, porque se requeriría un monto muy alto del valor marginal de la prima por producción intelectual, para equilibrar el tiempo durante el cual los profesores de la universidad pública, reciben su contraprestación, especialmente tratándose de profesores jóvenes.

Uno de los principales problemas a los que se enfrenta la universidad pública en Colombia en la contratación de nuevos profesores, son las restricciones legales que afronta para fijar un salario de enganche que pueda atraer a los candidatos más talentosos del mercado ${ }^{4}$.

4 En el año 2014, el salario promedio de enganche de un profesor con doctorado fue de apenas 3,8 millones de pesos en promedio. 
No obstante, la docencia en la universidad pública colombiana también tiene entre otros, un par de puntos a favor: i. No exige dedicación exclusiva, ii. Dependiendo de su antigüedad, experiencia $\mathrm{y}$ sobre todo publicaciones, un profesor de universidad pública puede llegar a ganar tanto o más que un profesor de universidad privada.

Si las ventajas que ofrece la universidad pública compensan el bajo salario de enganche de los profesores de la universidad pública, la distribución de habilidades investigativas de los profesores contratados no se vería afectada, y en ese caso podría afirmarse que la política de incentivos salariales es preferida a la de salarios altos.

En este sentido, políticas como el cargo de profesor de dedicación exclusiva pu- ede afectar positivamente la investigación, porque puede ayudar a reducir la restricción del salario de enganche que habilite la contratación de profesores con mayor habilidad para la investigación, ya que permite que el salario del profesor se incremente hasta en un $22 \%$. Sin embargo, no se esperaría un efecto directo del salario más alto sobre el esfuerzo a la investigación y la producción intelectual derivada.

La significancia y el efecto de las dummy de tiempo también dejaron ver el crecimiento ceteris paribus de la producción intelectual de los profesores de la Universidad del Valle, debido probablemente a efectos de cohorte y a la mayor oportunidad de publicación, asociada a una mejor oferta de revistas especializadas o mejor ranqueadas por Colciencias, o ambas situaciones.

\section{REFERENCIAS}

Aksnes, D., Rorstad, K., Piro, F. \& Sivertsen, G. (2011). Age and scientific performance. A largescale study of Norwegian scientists. In Paper presented at the ISSI 2011, Durban, South Africa.

Aksnes, D. W., Rorstad, K., Piro, F. \& Sivertsen, G. (2011b). Are female researchers less cited? A largescale study of Norwegian scientists. Journal of the American Society for Information Science and Technology, 62(4), 628-636.

Allison, P. \& Stewart, J. (1974). Productivity differences among scientists: evidence for accumulative advantage. American Sociological Review, 39, 596-606.

Archibugi, D. \& Coco, A. (2004). A new indicator of technological capabilities for developed and developing countries. World Development, 32(4), 629-654. 
Astin, H. \& Bayer, A. (1979). Pervasive sex differences in the academic reward system: Scholarship, marriage, and what else? In D.R. Lewis \& W.E. Becker (Eds.), Academic rewards in higher education. Cambridge, MA: Ballinger.

Baccini, A., Barabesi, L., Cioni, M. \& Pisani, C. (2014). Crossing the Hurdle: The determinants of individual scientific performance. Scientometrics, 1-28.

Bayer, A. \& Dutton, J. (1977). Career age and research-professional activities of academic scientists: tests of alternative nonlinear models and some implications for higher education faculty policies. The Journal of Higher Education, 48(3), 259-282.

Bonaccorsi, A. \& Daraio, C. (2003). Age effects in scientific productivity - the case of the Italian national research council (CNR). Scientometrics, 58(1), 49-90.

Carayol, N. \& Matt, M. (2006). Individual and collective determinants of academic scientists on productivity. Information Economics and Policy, 18, 55-72.

Carvajalino, A. \& Ariza, A. (2008). Evaluación de los incentivos a la investigación y extensión en la Universidad Industrial de Santander. Tesis de grado. Facultad de Ciencias Humanas. Escuela de Economía y Administración. Universidad Industrial de Santander.

Cohen, W., Florida, R. \& Goe, W. (1994). University-industry research centres in the United States. Pittsburgh, PA: Centre for Economic Development, Carnegie Mellon University.

Cole, S. (1979). Age and scientific performance. American Journal of Sociology, 84(4), 958-977.

Cole, J. \& Cole, S. (1973). Social stratification in science. Chicago: Chicago University Press.

Cole, S., Cole, J. \& Dietrich, L. (1978). Measuring the Cognitive State of Scientific Disciplines. In Y. Elkana, J. Lederberg, R. Merton, A. Thackray \& H. Zuckermann. (Eds.). Towards a metric of science: the advent of science indicators, (pp. 209-251). New York: Wiley.

Cole, J. \& Zuckerman, H. (1984). The productivity puzzle: Persistence and change in patterns of publication of men and women scientists. In M. W. Steimkamp \& M. Maehr (Eds.). Advances in motivation and achievement, 2, 217-258.

Crane, D. (1965). Scientists at major and minor universities: a study of productivity and recognition. American Sociological Review, 30(5), 699-714.

Creswell, J. W. (1986). Measuring faculty research performance. New Directions for Institutional Research 50. San Francisco: Jossey-Bass.

David, P. (1994). Positive feedbacks and research productivity in science: reopening another black box. In O. Grandstrand (Ed), Economics and Technology. Amsterdam: Elsevier. 
Salarios, incentivos y producción intelectual docente en la universidad pública ...

Jhon Alexánder Méndez Sayago - Leonardo Vera Azaf

Dundar, H. \& Lewis, D. (1998). Determinants of research productivity in higher education. Research in Higher Education, 39(6), 607-631.

Fielden, J. \& Gibbons, J. (1991). Merit myopia and business school faculty publications. Business Horizons, 34(2), 8-12.

Fox, M. (1992). Research, teaching, and publication productivity: mutuality versus competition in academia. Sociology of Education, 65(4), 293-305.

González, C. \& Veloso, F. (2007). The determinants of research output and impact: A study of Mexican researchers. Research Policy, 36, 1035-1051.

Goodwin, T. \& Sauer, R. (1995). Life cycle productivity in academics research: Evidence from cumulative publication histories of academic economists. Southern Economic Journal, 61(3), 728-743.

Gorbaneff, Y. (2003). Teoría del agente-principal y el mercadeo. Revista EAFIT, 129, 75-86.

Gutiérrez, J. \& Berrío, O. (2011). Punto de inflexión entre empresas y universidades ante la relación universidad, empresa y Estado en Colombia. Revista Universidad \& Empresa, 21, 167-191.

Guzmán, A. \& Trujillo, M. (2011). Políticas de incentivos relacionadas con la investigación: una revisión crítica desde la teoría de contratos. Estudios Gerenciales, 27(120), 127-145.

Jordan, J. M., Meador, M. \& Walters, S. (1989). Academic research productivity, department size and organization: further results. Economics of Education Review, 8(4), 345-352.

Knorr, K., Mittermeier, R., Aichholzer, G. \& Waller, G. (1979). Individual publication productivity as a social position effect in academic and industrial research units. In F. Andrews (Ed.), The effectiveness of research groups in six countries (pp. 55-94). Cambridge: Cambridge University Press.

Kyvik, S. (1991). Productivity in Academia. Scientific publishing at Norwegian universities. Oslo: Universitetsforlaget.

Kyvik, S. (1995). Are big university departments better than small ones? Higher Education, 30(3), 295-304.

Kyvik, S., \& Smeby, J. (1994). Teaching and research: The relationship between the supervision of graduate students and faculty research performance. Higher Education, 28(2), 227 239. 
Kyvik, S. \& Teigen, M. (1996). Child care, research collaboration, and gender differences in scientific productivity. Science, Technology \& Human Values, 21(1), 54-71.

Lehman, H. C. (1958). The chemist most creative years. Science, 127, 1213-1222.

Lehman, H. C. (1960). The decrement in scientific productivity. American Psychologist, 15, 128134.

Levin, S. \& Stephan, P. (1989). Age and research productivity of academic scientists. Research in Higher Education, 30(5), 531-549

Levin, S. \& Stephan, P. (1991) Research productivity over the life cycle: evidence for academic scientists. The American Economic Review, 81, 114-132.

Long, J. S. (1978). Productivity and academic positions in the scientific career. American Sociological Review, 43, 889-908.

Lotka A. (1926). The frequency distribution of scientific productivity. Journal of the Washington Academy of Sciences, 16, 317-323.

Manjarrés, L. (2009). Las relaciones universidad empresa y su efecto sobre la segunda misión universitaria. Tesis doctoral, Departamento de estadística e investigación operativa aplicada y calidad. Universidad Politécnica de Valencia

Merton, R.K. (1968). The Matthew effect in science. Science, 159(3810), 56-63.

Miller, A. \& Serzan, S. (1984). Criterion for identifying a refereed journal. Journal of Higher Education, 55(6), 763-699.

Obembe, O. (2012). Determinants of scientific productivity among Nigerian University academics. Indian Journal of Science and Technology, 5(2), 2155-2164.

Paasi, A. (2005). Globalization, academic capitalism, and the uneven geographies of international journal publishing spaces. Environment and Planning, 37, 769-789.

Perozo, S., De Arteaga, F. \& Fuenmayor, B. (2008). La productividad investigativa de los docentes del Instituto Universitario de Tecnología de Cabimas. Revista NEGOTIUM, 3(9), 72-87.

Piedra, Y. \& Martínez, A. (2007). Producción científica. Revista Ciencias de la información, 38(3), 33-38.

Porter, S. \& Umbach, P. (2001). Analyzing faculty workload data using multilevel modeling. Research in Higher Education, 42(2), 171-196. 
Salarios, incentivos y producción intelectual docente en la universidad pública ...

Jhon Alexánder Méndez Sayago - Leonardo Vera Azaf

Print, M. \& Hattie, J. (1997). Measuring Quality in Universities: An Approach to Weighting Research Productivity. Higher Education, 33(4), 453-469.

Rauber, M. \& Ursprung, H. (2008). Life cycle and cohort productivity in economic research: the case of Germany. German Economic Review, 9(4), 431-456.

Rebne, D. (1990). Determinants of individual productivity: a study of academic researchers. California: Institute of Industrial Relations Publications Center, UCLA.

República de Colombia (1992). Decreto 1444 de septiembre 3 de 1992. Por el cual se dictan disposiciones en materia salarial y prestacional para los empleados públicos docentes de las universidades públicas del orden nacional.

República de Colombia (2002). Decreto 1279 de Junio 19 de 2002. Por el cual se establece el régimen salarial y prestacional de los docentes de las Universidades Estatales.

Reskin, B. (1978). Scientific productivity, sex, and location in the institution of science. American Sociological Review, 83(5), 1235-1243.

Smeby, J. \& Try, S. (2005). Departmental contexts and faculty research activity in Norway. Research in Higher Education, 46(6), 593-619.

Stephan, P. (2010). The economics of science. In Handbook of the economics of innovation. Vol1, (pp 217-274). Burlington: Bronwyn H. \& Nathan Rosenberg, Acad. Press. .

Tang, C. (2010). A note on the nonlinear wages-productivity nexus for Malaysia. Munich, Germany: MPRA Paper, University Library of Munich.

Vella, F. \& Verbeek, M. (1997). Using rank order as an instrumental variable: an application to the return to schooling. CES Discussion Paper 97.10, K.U.: Leuven.

Weiss, Y. \& Lillard, L. (1982). Output variability, academic labor contracts, and waiting times for promotion. Research in Labor Economics, 5, 157-188.

Zuckerman, H. \& Merton, R. (1972). Age, aging, and age structure in science. In M. Riley, M. Johnson \& A. Foner (Eds.), A sociology of age stratification: Aging \& society (Vol. 3). New York: Russel Sage foundation.

Zuckerman, H. \& Merton, R. (1973). The Sociology of Science: Collected Papers of R.K. Merton, (Reimp.). Chicago, USA: Chicago University, Chicago Press. 